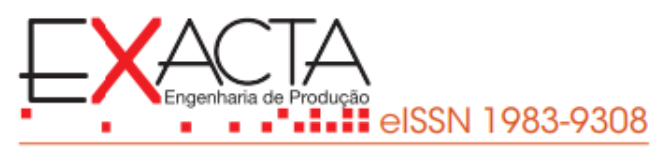

\title{
DETERMINAÇÃO DAS PRINCIPAIS CARACTERÍSTICAS DE AVALIAÇÃO DE AEROPORTOS HUB UTILIZANDO O MÉTODO AHP
}

\section{DETERMINATION OF MAIN HUB AIRPORT EVALUATION CHARACTERISTICS USING THE AHP METHOD}

Recebido em: 21 nov. 2019

Aprovado em: 13 maio 2020

Versão do autor aceita publicada online: 13 maio 2020

Publicado online: 19 maio 2021

\section{Como citar esse artigo - American Psychological Association (APA):}

Moura, I. R., Costa, L. H. G., Santos, E. M., \& Viana, H. R. G. (2021, out./dez). Determinação das principais características de avaliação de aeroportos hub utilizando o método AHP. Exacta. 19(4), 785-804. https://doi.org/10.5585/exactaep.2021.16066.

Submeta seu artigo para este periódico $\beta$

Dados Crossmark 


\title{
DETERMINAÇÃO DAS PRINCIPAIS CARACTERÍSTICAS DE AVALIAÇÃO DE AEROPORTOS HUB UTILIZANDO O MÉTODO AHP
}

\author{
DETERMINATION OF MAIN HUB AIRPORT EVALUATION CHARACTERISTICS USING \\ THE AHP METHOD
}

\begin{abstract}
iD Ingrid Rebouças de Moura ${ }^{1}$
iD Luis Henrique Gonçalves Costa ${ }^{2}$

iD Enilson Medeiros dos Santos

(iD)

Herbert Ricardo Garcia Viana ${ }^{4}$
\end{abstract}

\author{
${ }^{1}$ Mestre \\ Universidade Federal do Rio Grande do Norte - UFRN. \\ Natal, Rio Grande do Norte - Brasil. \\ ingrid.moura.062@ufrn.edu.br \\ ${ }^{2}$ Mestre \\ Universidade Federal de Pernambuco - UFPE. \\ Universidade Federal Rural do Semi-Árido - UFERSA. \\ Natal, Rio Grande do Norte - Brasil. \\ luis.henrique@ufersa.edu.br \\ ${ }^{3}$ Doutor \\ Universidade Federal de Pernambuco - UFPE. \\ Natal, Rio Grande do Norte - Brasil. \\ enilsonsantos451@gmail.com \\ ${ }^{4}$ Doutor \\ Universidade Federal do Rio Grande do Norte - UFRN. \\ Natal, Rio Grande do Norte - Brasil. \\ herbertviana@ct.ufrn.br
}

Recebido em: 21 nov. 2019

Aprovado em: 13 maio 2020

\section{Introduçào}

Resumo: A escolha de um aeroporto hub por uma empresa aérea garante uma série de benefícios relacionados à movimentação de passageiros, o que implica em um estudo detalhado quanto a demanda, localização geográfica e infraestrutura aeroportuária disponível para acomodar esse novo modelo de operação de voos. Neste sentido, este artigo tem como objetivo realizar um estudo quanto às principais características para que um aeroporto se estabeleça como um hub, firmando a importância que um componente tem sobre outro, como fator de instalação do sistema, na visão de especialistas. A metodologia da pesquisa está baseada na utilização do Analytical Hierarchy Process (AHP) para determinar a relevância dos componentes para escolha de um aeroporto hub. O resultado do trabalho caracteriza os principais indicadores e determina os critérios mais relevantes para a avaliação do potencial de um dado aeroporto para abrigar operações de hub.

Palavras-chave: Aeroporto hub. Operações aeroportuárias. Multicritério. AHP.

Abstract: The choice of a hub airport by an airline ensures a number of benefits related to the movement of passengers, which implies the needs for detailed studies on demand, geographical location and airport infrastructure available to accommodate the new model of flight operations. In this sense, this article aims to analyze the main characteristics for an airport to establish as a hub, assessing the importance that one criterium has over another, as a factor of system installation, in the view of specialists. The research methodology is based on the use of the Analytical Hierarchy Process (AHP) to determine the weight of components for an airport hub. Results characterize the main criteria and determine the most relevant ones for the evaluation of the potential of a given airport to receive hub operations.

Keywords: Hub airport. Airport operations. Multicriteria. AHP. 
Um aeroporto tem como função principal comportar a movimentação de passageiros e cargas, podendo ser avaliado em termos de eficiência quanto a frequência de voos oferecidos. Segundo Ashford, Stanton, Moore, Coutu e Beasleyet (2015), as empresas aéreas passaram a possuir maior controle dos serviços de rotas e frequências logo após a desregulamentação, o que possibilitou a instalação dos aeroportos hubs. A instalação de redes hub and spoke permite às companhias aéreas a redução de seus custos de operação de aeronaves e a redução de atrasos no cronograma dos passageiros, uma vez que atinge maiores fatores de carga em aeronaves maiores com maior frequência de serviço. Esse sistema permite combinar passageiros com a mesma origem, mas com diferentes destinos no mesmo voo e vice-versa, podendo por meio deste sistema ampliar o número de passageiros quando comparado a modelos de ponto a ponto (Daniel, 1995; Lin, 2006).

A escolha de um hub por uma empresa aérea envolve um alto investimento para o qual se espera um retorno com o aumento de eficiência dos voos realizados e dos serviços prestados. Logo, essa decisão engloba uma série de fatores não somente relacionados à demanda econômica ou posição geográfica, bem como referentes à infraestrutura e às operações do aeroporto (Pishdar, Ghasemzadeh \& Antuchevičienè, 2019; Mohri, Karimi, Kordani \& Nasrollahi, 2018; Torkestani, Seyedhosseini, Makui \& Shahanaghi, 2018; Yang \& Chiu, 2015; Ssamula, 2010).

Diante do que foi abordado na revisão de literatura e da busca por caracterizar esses aeroportos em questão, justifica-se a realização do estudo na área de aeroportos hubs pela escassez de trabalhos que apresentem indicadores relevante para as empresas aéreas no momento de decisão de escolha de um hub, como também determinar uma ordenação para mais bem avaliar esses critérios dentro de um aeroporto. A seleção de indicadores também pode incentivar a competitividade entre aeroportos, elevando os níveis de operações. Desta maneira, este artigo busca caracterizar e definir por meio do método de apoio a decisão multicritério, Analytical Hierarchy Process (AHP), indicadores relacionados à infraestrutura aeroportuária - Lado Ar, Lado Terra e Serviços de Suporte -, que norteiam a escolha de um aeroporto hub. Portanto, o estudo busca definir a importância relativa e a relevância desses critérios para melhorar a escolha por parte das empresas aéreas.

O presente trabalho está assim estruturado: (2) Breve revisão de literatura contendo a caracterização de um aeroporto hub, (3) metodologia empregada para elaboração e aplicação dos questionários da pesquisa, apresentação da escala de prioridades relativas do método e seleção dos indicadores analisados, (4) análise dos dados e resultados do grau de importância das variáveis do aeroporto e (5) apresentação das considerações finais.

\section{Revisão de literatura}


Um aeroporto pode ser entendido como um sistema responsável por atender necessidades relacionadas ao movimento de pessoas e mercadorias, necessidades essas que vêm apresentando grande crescimento nas últimas décadas, o que pode estar associado ao desenvolvimento do turismo e à consolidação dos processos globais de manufatura e comércio de mercadorias. Por outro lado, a infraestrutura aeroportuária tem passado por grandes mudanças em consequência das alterações no mercado de transporte aéreo (Almeida, 2011).

O aeroporto é o componente principal no transporte aéreo, devendo sua estrutura física comportar a transferência modal entre os sistemas aéreos e terrestres. Logo, é um importante sistema de interação dos três maiores elementos que compõem o transporte aéreo: o próprio sistema aeroportuário e seus sistemas de controle, as companhias aéreas e os usuários. Ashford et al. (2015) afirmam que um aeroporto pode ser entendido como um sistema que se divide de acordo com seus serviços operacionais: Lado Ar e Lado Terra. O Lado Ar é composto pelo espaço aéreo em torno do aeroporto, compreendendo as pistas de circulação, oferece acomodação para a chegada de aeronaves antes do pouso e de aeronaves de partida logo após a decolagem. Por conseguinte, o sistema referente ao Lado Terra abrange os sistemas de acesso à superfície, ligando o aeroporto à sua área de captação e ao sistema de terminais de passageiros e cargas. Segundo Moreira (2006), um aeroporto tem como objetivo garantir que as transferências modais sejam executadas de forma segura e ordenada, de modo que sua infraestrutura permita aos usuários o conforto durante o aguardo de suas atividades. Da mesma forma, deve possuir a capacidade de se expandir e possuir flexibilidade suficiente para atender todas as oscilações com relação a demanda.

Senguttuvan (2006) explica a visão sinalizada pela moderna teoria de gestão de redes aeroportuárias, em que um aeroporto pode se estabelecer como um "Centro de Negócios Multimodal", uma vez que o desenvolvimento de aeroportos de sucesso auxilia no incentivo a formação de grupos de indústrias, além de contribuir na geração de milhões de oportunidades de emprego em todo o mundo, e por fim, ser uma vitrine de realizações culturais, econômicas e tecnológicas da nação.

Nas últimas décadas, é perceptível o crescimento do setor da aviação civil. Em janeiro de 2017, a OACl - Organização da Aviação Civil Internacional divulgou que houve 35 milhões de embarques em todo o mundo em 2016, com o número de passageiros pagantes-quilômetro (RPK) chegando a 7,015 bilhões (Addepalli, Pagalday, Salonitis \& Roy 2018). Nos aeroportos brasileiros, a movimentação de passageiros (pax) dobrou entre 2006 e 2015, atingindo 200 milhões/ano (Costa \& Santos, 2016).

O crescimento acelerado na demanda aérea provocou aumento nos atrasos das viagens, além de congestionamentos (Zhang \& Czerny, 2012; Gillen, Jacquillat \& Odoni, 2016). Mesmo com a crescente demanda na construção de novos aeroportos e pistas de pouso e decolagem (PPD), persiste em alguns aeroportos a falta de capacidade. Por outro lado, cresce a importância das receitas 
alternativas ligadas a pistas, estacionamentos e terminais aeroportuários, tornando-se comum a adesão às receitas de serviços não aeronáuticos, que incluem as lojas comerciais, publicidade, locação de carros, estacionamento e aluguel de áreas dentro do sítio aeroportuário (Kidokoro \& Zhang, 2018).

A utilização de aeroportos hub pode reduzir os atrasos de congestionamentos, porém aumenta o atraso de parada (Daniel, 1995). A instalação do sistema hub and spoke só foi possível para as empresas aéreas em decorrência da desregulamentação, que se concluiu por volta de 1997, resultando na ampliação do setor e na alteração da dinâmica dos serviços de transporte aéreo. Por meio desta nova reestruturação, se passou a adotar o sistema hub and spoke como estratégia para melhorar a frequência de voos.

\subsection{Sistematização de um aeroporto hub}

Segundo Button (2002), não há uma definição única ou amplamente usada para um aeroporto hub, seja ela de um ponto de vista econômico ou legal, pois todas elas refletem limitações práticas da qualidade dos dados e informações disponíveis, ou considerações normativas sobre a natureza do argumento sendo favorecido. Logo, será apresentada a definição mais geral em termo da forma e da funcionalidade desses aeroportos de acordo com o que é demonstrado na literatura.

Para melhor entendimento, pode-se diferenciar as duas formas de ligação, hub and spoke e ponto a ponto, pela observação da Figura 1. Nas rotas ponto a ponto, as ligações ocorrem somente entre dois pontos (Figura 1.a), já nas rotas Hub and Spoke, os pontos são ligados a um ponto central, como apresentado abaixo (Figura 1.b), importando em um aumento da malha (Lelles, 2001).

\section{Figura 1}

Tipos de rotas

a)

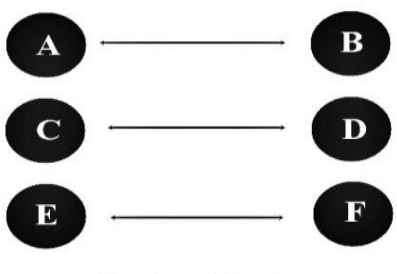

Ponto a Ponto b)

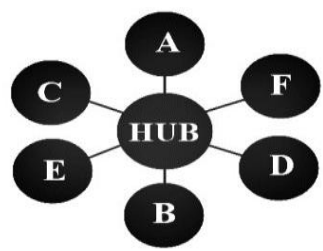

Hub and Spoke

Fonte: Adaptada de Lelles, 2001

De acordo com Lin (2006), em uma rede simples ligando um aeroporto hub e dois aeroportos spokes, o aeroporto hub está em uma posição de monopólio, no qual o desenvolvimento das companhias de redes aéreas torna-se possível pelo preenchimento de janelas horárias por parte do hub que realiza a ligação entre estes aeroportos spoke. Neste caso, o aeroporto hub, na verdade, disputa 
contra um outro hub potencial no mercado de voos de ligação. Assim, devido ao crescimento dramático de redes hub and spoke, a competição entre esses aeroportos centrais tem se tornado cada vez mais frequentes em muitos países do mundo.

Conforme Graham (2014) a competição tradicional entre aeroportos é uma competição entre companhias aéreas. Os aeroportos próximos competem para atrair serviços aéreos para seus usuários em que as viagens se originam ou terminam na região. As redes hub and spoke deram origem a uma segunda forma de competição entre aeroportos, que podem estar localizados em diferentes regiões para oferecer serviços de hub, com voos de diferentes origens para o mesmo destino ou da mesma origem para diferentes destinos, e que são concentrados passando por nós intermediários (os hubs).

\subsection{Operações de um aeroporto hub}

As companhias aéreas são responsáveis por ajustar as chegadas e partidas de forma que os horários dos mais diversos voos de ligação sejam coordenados e que os usuários não percam muitas horas de espera ao aguardo do seu destino final (Almeida e Costa, 2014; Danesi e Lupi, 2005). O que acontece é que há passageiros que voam de diferentes origens em direção a destinos que apresentam pouca demanda para voos diretos: logo, se faz necessário que os passageiros saiam de um aeroporto considerado como spoke e efetuem uma escala em um aeroporto hub, onde são transferidos para outra aeronave que os transporta até o seu destino.

Redondi, Malighetti e Paleari (2011) explicam o hub and spoke como um sistema simples composto de dois aeroportos "spoke", um A e outro B, os quais se conectam entre si apenas por meio de um terceiro aeroporto central, $\mathrm{H}$, sendo esse responsável por deter o monopólio do mercado (Figura 2). Nesse caso, pode-se afirmar que a pressão exercida por alianças e operadoras independentes podem gerar mais de uma opção para conexão entre qualquer par de aeroportos.

\section{Figura 2}

Esquema de operações hub and spoke

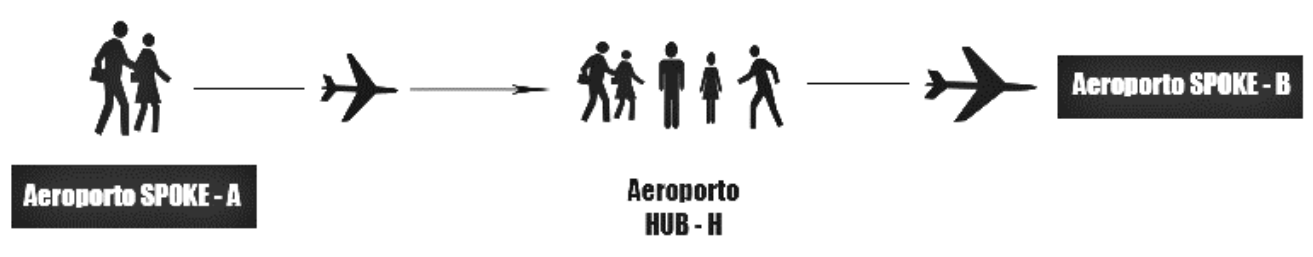

Fonte: Autores. 
Um passageiro normalmente faz sua escolha quanto à empresa aérea de acordo com indicadores de frequência, preço e demais critérios que dizem respeito à qualidade esperada do serviço. Todavia, tais critérios podem ser resumidos em três fatores principais: a conectividade que é oferecida, pois o passageiro tem o desejo de chegar a seu destino final o mais rápido possível; o custo total da viagem, definido principalmente pelas tarifas de voo; e a qualidade do serviço em si, que irá englobar a pontualidade, presença de serviços auxiliares e congestionamento no aeroporto (Redondi et al., 2011).

De acordo com Button, Lall, Stough e Trice (1999), passageiros com viagens a negócio são menos sensíveis aos custos tarifários, mas exigem maior qualidade do serviço, em termos de tempo e frequência de voos, instalações de lazer e outros. Isso reflete a importância dos custos generalizados em suas tomadas de decisão. Ao oferecer entradas flexíveis, conforto, comodidade e agendamento de voos convenientes às necessidades de negócios a um preço premium, as operadoras podem atrair esses usuários.

Segundo Borenstein (1989), que estudou os fatores econômicos e a dinâmica competitiva que levam os operadores a escolherem um sistema hub and spoke, um sistema como este utiliza um número menor de voos para conectar cada nó da rede do sistema. Isso leva a um uso mais eficiente dos recursos de transporte, permitindo que companhias aéreas empreguem aeronaves maiores e em frequências mais altas, obtendo economias de densidade.

Uma rede hub and spoke é uma estratégia orientada por fornecedores, que potencializa as conexões entre as mesmas operadoras disponíveis para uma determinada companhia aérea em um aeroporto hub. Logo, a configuração Hub and Spoke permite se chegar a uma rede na qual voos diretos são substituídos por um número mais elevado de conexões indiretas (Song \& Ma, 2006).

Ainda por Song e Ma (2006), o objetivo principal de um hub em uma companhia aérea é aumentar o número de possibilidades de viagens que podem ser realizadas pela companhia. Para isso, é preciso contar com uma agenda cuidadosa que será essencial para vincular o maior número possível de pares de cidades, a fim de diminuir o tempo que passageiros aguardam no aeroporto, logo, envolvendo um grande número de aeronaves que chegaram a determinado aeroporto hub em um curto espaço de tempo, seguida por uma demanda semelhante de partidas.

As operações hub and spoke geram benefícios tanto para as companhias aéreas como para os passageiros. Para Button et al. (1999), que pesquisaram os ganhos conquistados pelas companhias em termos de economias de escala, escopo e densidade, dando atenção à demanda e às economias relevantes no mercado, há um forte envolvimento de implicações positivas que uma grande rede pode ter no patrocínio e na receita obtida por uma operadora. A análise destes autores observou, por exemplo, que devido a economias de densidade, um aumento de $1 \%$ no número de passageiros transportados por uma companhia aérea resultou em uma redução de $0,8 \%$ nos custos totais. 
Além da diversidade de fatores positivos quanto a infraestrutura, atendimento e economia, é importante salientar os benefícios gerados para a cidade que comporta um aeroporto central. Conforme Button et al. (1999), aqueles que residem em cidades com aeroporto hub têm certas vantagens. A seguir são apresentadas algumas das características que categorizam a cidade hub quando comparadas a áreas urbanas com aeroportos que possuem outras formas de serviços de transporte aéreo:

- Maior frequência de voos;

- Voos diretos - segundo Button et al. (1999), uma pesquisa de 1996 salientou, controlado o tamanho da população, que os aeroportos hub eram capazes de oferecer voos sem escalas para quase o dobro de cidades, com 25\% a mais de saídas diárias por cidade atendida;

- Mais possibilidades de voos com retorno no mesmo dia;

- Probabilidade maior de voos internacionais;

- Serviços voltados às necessidades do mercado local, como possibilitar destinos atraentes aos residentes;

- Os moradores de cidades hub possuem a oportunidade de se conectarem a outros grandes centros.

A FAA (Federal Aviation Administration) argumenta que moradores próximos a aeroportos centrais têm o hub como um benefício, pois muitos voos sem escalas estão disponíveis para muitas cidades que de outra forma não seriam capazes de suportar tal serviço. Em termos econômicos, isso quer dizer que essas regiões desfrutam de benefícios externos de ter passageiros em trânsito passando por seu aeroporto local que thes permitem acesso a uma grande rede de transporte aéreo regular (Button et al., 1999).

Por fim, segundo Campos, Silva, Pereira e Moreira (2010), podemos chegar à conclusão de que os aeroportos hub são essenciais para a produção e distribuição, o que faz com que tenham papel fundamental na promoção do desenvolvimento das cidades, atraindo empresas e originando negócios em suas proximidades.

\section{Metodologia}

O presente artigo caracteriza-se por uma pesquisa teórica-empírica, em que a revisão de literatura oferece suporte teórico na construção das discussões e ideias transmitidas, e a pesquisa empírica desenvolve-se na forma de uma investigação sobre um fenômeno contemporâneo dentro de seu contexto real: neste caso, a investigação dos indicadores para alocação de aeroportos hubs com especialistas da área. 
Após a revisão da literatura e obtidos os procedimentos para aplicação do método AHP, o passo seguinte seria montar a estrutura do modelo a ser avaliado, como é apresentado na Figura 3. O aplicativo utilizado para montar a matriz de julgamento e avaliar a consistência dos valores determinados foi o sistema online BPMSG (Business Performance Management Singapore) AHP, implementado e descrito em Goepel (2018). De livre acesso na web, este sistema calcula automaticamente a razão de consistência (CR) através da ferramenta AHP Priority Calculator, e mostra algumas opções para melhorar a consistência das alternativas, além de possibilitar e otimizar o tempo quando trabalhado com um grande número de critérios de avaliação.

Figura 3

Estrutura hierárquica do modelo de decisão

OBJETIVO

Grau de Importância dos Componentes de um Aeroporto Hub

Fonte: Autores.

O método utilizado determina o peso dos critérios e seus valores de desempenho nas alternativas por meio da utilização de matrizes de julgamento, que nesta pesquisa serão construídas pelo AHP Priority Calculator. Na prática, cada critério será avaliado e classificado por grau de importância: para isso elaborou-se um questionário de opinião com o auxílio do Google Docs, enviado a seis especialistas de gestão de infraestrutura aeroportuária (das áreas de operações, segurança, comercial, manutenção e segurança operacional).

\subsection{O método AHP (Analytical Hierarchy Process)}

O método AHP é um processo hierárquico na medida que em seu desenvolvimento realiza comparações a partir de uma escala fundamental que represente a preferência, tendo a preocupação de obter resultados consistentemente aferidos, tanto internamente aos grupos de elementos de sua estrutura, quanto entre eles. Segundo o autor, o método tem amplas aplicações na tomada de decisão, planejamento e alocação de recursos e na resolução de conflitos (Saaty, 1987).

Saaty (1987) apresenta a formação da estrutura hierárquica de um problema como um desmembramento a partir de um foco principal, que seria o objetivo geral, até seus critérios e a 
subdivisão destes em subcritérios até finalmente chegar as alternativas que iram solucionar o problema inicial. Nosal e Solecka (2014) descrevem que todas as variáveis em um problema são comparáveis no método, significa que em cada par de variantes, o decisor sempre terá uma preferência ou irá considerálas equivalentes. A modelagem de preferência é feita comparando pares de critérios ou subcritérios. Conforme Lambert (1991), o processo de decisão hierárquico de Saaty classifica as alternativas discretas usando a comparação paritária das alternativas, no intuito de classificar $n$ alternativas.

As decisões relacionando múltiplos critérios constituem uma área de conhecimento resultante da investigação, que fornece a quem cabe o direito de decisão as ferramentas e os métodos (no caso, o método AHP) que ajudam a resolver problemas complexos de decisão, considerando pontos de vista, que muitas vezes podem ser conflitivos.

Um problema de decisão multicritério pode envolver: a Seleção (otimização), onde se tem um subconjunto de decisões (ação, opções) consideradas as melhores em termos da família de critérios considerada; a Classificação (ordenação), em que o tomador de decisão divide um conjunto de decisões (atividades e variantes de ação) em subconjuntos (classes, categorias), de acordo com os padrões aceitos; e, a Hierarquia (ranking), quando o tomador de decisão visa colocar as alternativas em ordem decrescente (Nosal \& Solecka, 2014).

Portanto, o AHP tem a vantagem de avaliar um conjunto de critérios em uma estrutura hierárquica, permitindo que o usuário tenha um foco melhor ao alocar os pesos aos critérios e subcritérios. Essa etapa é importante, porque uma estrutura diferente pode levar a uma classificação final diferente. Ao configurar a hierarquia do AHP com um grande número de elementos, o tomador de decisão deve tentar organizar esses elementos em grupos em que eles não sejam diferentes de maneiras extremas (Franek \& Kresta, 2014).

Existem situações em que o número de variáveis é demasiadamente alto e é preciso fazer subdivisões do que será analisado. É interessante que cada critério ou subcritério pertença a um grupo no qual todos os elementos tenham características compatíveis, a fim de garantir maior precisão na avaliação par a par, à qual os psicólogos atribuem a obtenção de respostas mais precisas quando comparadas a análises em conjunto (Bandeira \& Correia, 2006).

Nesse contexto, a utilização de um mecanismo online para aplicação da estrutura do método AHP (BPMSG) possibilita agilidade na construção dos pesos, além da vantagem em sugerir as modificações necessárias ao decisor para que as respostas adquiram consistência dentro da metodologia (Goepel, 2018). 


\subsection{Seleção dos componentes aeroportuários}

Os componentes adotados na avaliação foram selecionados com base na literatura relacionada com o sistema hub and spoke de acordo com sua operação e discutida com especialistas que listaram os principais indicadores como apresentado na Tabela 1 , que mostra o conjunto de critérios e subcritérios que serão analisados. Ressalta-se que determinados critérios serão facilmente encontrados na literatura e outros fazem parte da opinião conjunta dos especialistas consultados.

\section{Tabela 1}

Critérios e subcritérios de avaliação de um aeroporto hub

\begin{tabular}{|c|c|c|}
\hline Critério 1: Lado Ar & Critério 2: Lado Terra & Critério 3: Serviços de Suporte \\
\hline $\begin{array}{l}\text { Pista de Pouso e Decolagem } \\
\text { (PPD) }\end{array}$ & Check-in exclusivo & Modos de acesso \\
\hline Pistas de Táxi & Check-in compartilhado & $\begin{array}{l}\text { Confiabilidade do tempo de } \\
\text { acesso }\end{array}$ \\
\hline Auxílios de Navegação Aérea & $\begin{array}{l}\text { Área de processamento de } \\
\text { bagagens (bag drop) }\end{array}$ & Disponibilidade de Hotéis (leitos) \\
\hline $\begin{array}{l}\text { Instrumentos de Navegação } \\
\text { Aérea }\end{array}$ & Dimensão do Saguão & $\begin{array}{l}\text { Disponibilidade de Hotéis } \\
\text { (distância) }\end{array}$ \\
\hline $\begin{array}{l}\text { Número de Classificação de } \\
\text { Proteção Requerida (NCPR) }\end{array}$ & $\begin{array}{l}\text { Mix de Estabelecimentos } \\
\text { Comerciais }\end{array}$ & $\begin{array}{l}\text { Disponibilidade de Locais para } \\
\text { realização de Eventos }\end{array}$ \\
\hline $\begin{array}{l}\text { Número de Classificação de } \\
\text { Proteção Existente (NCPE) }\end{array}$ & Praça de Alimentação & \\
\hline $\begin{array}{l}\text { Capacidade de Abastecimento } \\
\text { (PAA) }\end{array}$ & $\begin{array}{l}\text { Canais de Inspeção de } \\
\text { Segurança }\end{array}$ & \\
\hline $\begin{array}{l}\text { Capacidade de Pouso e } \\
\text { Decolagem (slots) }\end{array}$ & Área da Sala de Embarque & \\
\hline \multirow[t]{8}{*}{$\begin{array}{l}\text { Disponibilidade de Área para } \\
\text { Manutenção de Aeronaves }\end{array}$} & $\begin{array}{l}\text { Pontes de } \\
\text { Embarque/Desembarque }\end{array}$ & \\
\hline & Embarque Remoto & \\
\hline & $\begin{array}{l}\text { Número de Esteiras de } \\
\text { Desembarque }\end{array}$ & \\
\hline & $\begin{array}{l}\text { Sistema de informação ao } \\
\text { passageiro }\end{array}$ & \\
\hline & Facilidade de Conexão & \\
\hline & Disponibilidade de Meio-Fio & \\
\hline & $\begin{array}{l}\text { Área do Estacionamento } \\
\text { Comercial }\end{array}$ & \\
\hline & $\begin{array}{l}\text { Disponibilidade de Hotel de } \\
\text { Passagem }\end{array}$ & \\
\hline
\end{tabular}

Fonte: Autores.

Os indicadores selecionados são exemplificados a seguir.

Lado $\mathrm{Ar}$

- Pista de Pouso e Decolagem: Área retangular, definida em um aeródromo em terra, preparada para pousos e decolagens de aeronaves; 
- Pistas de Táxi: Trajetória definida em um aeródromo em terra, estabelecida para táxi de aeronaves e com a função de oferecer uma ligação entre as partes do aeródromo;

- Auxílios de Navegação Aérea: Dispositivo externo a uma aeronave destinado a auxiliá-la na determinação de sua posição ou de um rumo seguro, ou ainda, alertá-la de perigos ou obstruções;

- Instrumentos de Navegação Aérea: Instrumentos capazes de conduzir um veículo voador de um lugar a outro em segurança, piloto faz sem necessitar enxergar o que está acontecendo fora do avião;

- Número de Classificação de Proteção Requerida (NCPR): Classificação que se baseia no grau de risco peculiar às operações do aeródromo, e que corresponde à categoria do mesmo para fins de prevenção, salvamento e combate a incêndio em aeronaves;

- Número de Classificação de Proteção Existente (NCPE): Classificação numérica que se baseia nos recursos humanos e materiais, existentes e disponíveis no aeródromo, para fins de prevenção, salvamento e combate a incêndio em aeronaves.

\section{Lado Terra}

- Check-in Exclusivo: Balcão da companhia aérea, ou da agência de viagens para compra de passagens, apresentação de documentos, despacho de bagagens e retirada do cartão de embarque;

- Check-in Compartilhado: Balcão que engloba várias companhias para a realização do checkin.

- Área de Processamento de Bagagens (bag drop): Espaços e instalações destinados ao manuseio da carga aérea, incluindo pátios de aeronaves, terminais de carga, armazéns, estacionamento de veículos e vias de acesso adjacentes;

- Dimensão do Saguão: Sala de entrada coberta, onde começa a escadaria ou ficam os elevadores que levam aos andares superiores;

- Mix de Estabelecimentos Comerciais: Lojas com estruturas dentro aeroporto, para atender os passageiros.

- Praça de Alimentação: Local destinado para o consumo de alimentos, usualmente no sistema de comida rápida (fast food);

- Canais de Inspeção de Segurança: Locais destinados a procedimentos de inspeção no embarque dos passageiros;

- Área da Sala de Embarque: Espaço onde o passageiro aguarda seu voo e realiza todos os procedimentos necessários para sua segurança e conforto; 
- Pontes de Embarque/Desembarque: Equipamento que faz a conexão entre o terminal de passageiros (TPS) e a porta da aeronave, possibilitando o embarque e desembarque;

- Embarque Remoto: Disponibilidade de ônibus que transportem os passageiros da sala de embarque até a aeronave;

- Número de Esteiras de Desembarque: Quantidade de transportadores de esteira, utilizado para transportar bagagens e cargas da unidade de transferência no solo para o porão da aeronave ou vice-versa;

- Sistema de Informação ao Passageiro: Informações gerais, placas direcionais, áreas de telefone, meios que permitem a informação necessária para o passageiro realizar os procedimentos adequados antes da saída do voo;

- Facilidade de Conexão: Facilidade que o passageiro possui em fazer conexão com outro voo.

- Disponibilidade de Meio-Fio: Área de meio fio (calçada) para embarque e desembarque de passageiros;

- Área do Estacionamento Comercial: Área destinada a veículos de conexão rápida.

- Disponibilidade de Hotel de Passagem: Quantidade de hotéis disponíveis para passageiros em conexão.

\section{Serviços de Suporte}

- Modos de Acesso: Modos que permitem acesso ao aeroporto (locomoção).

- Confiabilidade do Tempo de Acesso: Facilidade de locomoção com conexão aeroporto-centro, a qualquer momento.

- Disponibilidade de Hotéis (leitos): Quantidade de hotéis com leitos disponíveis na localidade do aeroporto.

- Disponibilidade de Hotéis (distância): Quantidade de hotéis disponíveis nas proximidades do aeroporto.

- Disponibilidade de Locais para realização de Eventos: Quantidade de áreas destinadas a lazer e entretenimento, disponíveis na proximidade do aeroporto.

A escala fundamental de Saaty poderia ser de difícil compreensão no momento de aplicação dos questionários, por isso se adotou a sua relação com uma escala percentual proposta por Bandeira e Correia (2006) no intuito de facilitar a coleta de dados por parte dos especialistas na área. Assim, foi possível realizar a comparação por pares quanto à importância de um componente ou indicador sobre outro. A Tabela 2 apresenta a relação entre as escalas utilizadas. É importante salientar que Bandeira e Correia (2006) definem com esta escala que todos os critérios são importantes, por isso não se consideram valores de 100\%/0\%. 
Tabela 2

Relação da escala utilizada com a Escala de Saaty

\begin{tabular}{|c|c|c|c|}
\hline \multicolumn{2}{|c|}{$\begin{array}{c}\text { Escala } \\
\text { Percentual } \\
\text { (\%) }\end{array}$} & $\begin{array}{c}\text { Escala } \\
\text { Fundamental de } \\
\text { Saaty }\end{array}$ & Grau de Importância \\
\hline$x$ & Y & & Definição \\
\hline $90 \%$ & $10 \%$ & 9 & Componente $\mathrm{X}$ é extremante mais importante que o Componente $\mathrm{Y}$. \\
\hline $80 \%$ & $20 \%$ & 7 & Componente X é muito importante em relação ao Componente Y. \\
\hline $70 \%$ & $30 \%$ & 5 & Componente X é importante em relação ao Componente Y. \\
\hline $60 \%$ & $40 \%$ & 3 & $\begin{array}{l}\text { Componente X é um pouco mais importante em relação ao Componente } \\
\text { Y. }\end{array}$ \\
\hline $50 \%$ & $50 \%$ & 1 & Os dois Componentes têm a mesma importância. \\
\hline $40 \%$ & $60 \%$ & $1 / 3$ & $\begin{array}{l}\text { Componente } Y \text { é um pouco mais importante em relação ao Componente } \\
\text { X. }\end{array}$ \\
\hline $30 \%$ & $70 \%$ & $1 / 5$ & Componente $Y$ é importante em relação ao Componente $X$. \\
\hline $20 \%$ & $80 \%$ & $1 / 7$ & Componente $\mathrm{Y}$ é muito importante em relação ao Componente $\mathrm{X}$. \\
\hline $10 \%$ & $90 \%$ & $1 / 9$ & Componente $\mathrm{Y}$ é extremante mais importante que o Componente $\mathrm{X}$. \\
\hline
\end{tabular}

\subsection{Avaliação dos dados obtidos}

A coleta de dados é o maior desafio em uma pesquisa de opinião, embora as respostas viessem de especialistas, o questionário proposto foi analisado individualmente no intuito de identificar a incoerência de determinadas respostas, que identificada, aconselha-se a proposta de uma nova análise por parte do respondente. Desta forma, foram elaboradas matrizes com os dados obtidos de cada especialista, e avaliado o CR - razão de consistência -, verificando a consistência das respostas. De acordo com o método, as respostas podem ser consideradas consistentes quando possuem $\mathrm{CR}<10 \%$.

\section{Análise dos dados e resultados}

Com os resultados de cada matriz, elaborada de acordo com os dados obtidos das respostas dos especialistas, o cálculo do peso da importância do indicador foi obtido com a média aritmética dos resultados apresentados em cada matriz.

A Tabela 3 apresenta os pesos referentes aos critérios adotados, que indicam o grau de importância para os especialistas em relação as áreas de estudo: Lado $\operatorname{Ar}$ (LA), Lado Terra (LT) e os Serviços de Suporte (SS). De acordo com a Tabela 3, o Lado Ar (52,45\%) é o componente mais importante, a partir das matrizes de julgamento que alcançaram consistência em torno de 9,55\%. Esse resultado transmite a importante da capacidade de operações com aeronaves, já estudada por Mohri, Karimi, Kordani e Nasrollahi (2018) na determinação da localização de hubs por meio de um modelo considerando a chegada e partida de voos, uma vez que a principal finalidade deste tipo de sistema é o aumento de conexões, Meson-mancha, Lange, Koelle \& Carro (2019) ressaltam que a capacidade do Lado Ar é um fator determinante na evolução do setor aéreo. Os Serviços de Suporte, mesmo ocupando 
o último nível, tem grau de importância próximo ao do Lado Terra, isso provavelmente porque ambos têm preocupação com o atendimento aos usuários.

\section{Tabela 3}

Grau de importância entre áreas de um aeroporto

\begin{tabular}{|l|l|l|}
\hline \multicolumn{1}{|c}{ Áreas } & Prioridade & Rank \\
\hline 1. Lado Ar (LA) & $52,45 \%$ & 1 \\
\hline 2. Lado Terra (LT) & $27,55 \%$ & 2 \\
\hline 3. Serviços de Suporte (SS) & $20,00 \%$ & 3 \\
\hline Fonte: Autores. & & \\
\hline
\end{tabular}

Com base nos dados, e de forma a avaliar a infraestrutura aeroportuária quanto à implantação de um aeroporto hub, utilizando a escala Likert na avaliação do indicador, pode-se adotar a Equação 1.

$$
\text { NOTA DO AEROPORTO }=0,5245 \mathrm{LA}+0,2755 \mathrm{LT}+0,20 \mathrm{SS}
$$

A Tabela 4 apresenta os dados relativos aos indicadores do Lado Ar, com razão de consistência 7,65\%, onde a maior importância está relacionada com a Pista de Pouso e Decolagem (PPD), seguido pelas Pistas de Táxi, pelos Números de Classificação de Proteção Existente (NCPE) e Requerida (NPCR), destacando sua importância, visto que influenciam na operação aeroportuária restringindo ou autorizando o pouso e decolagem de aeronaves. Posteriormente, aparecem a Capacidade de Pouso e Decolagem (slots), Auxílios e Instrumentos de Navegação Aérea. Destaque-se que os indicadores com maiores prioridades se referem aos principais ativos de transferência entre o transporte terrestre e o aéreo, e os responsáveis por gerar maior número de slots. Trata-se de resultado pertinente quando uma das maiores preocupações atualmente está no congestionamento (Zhang \& Czerny, 2012; Yang \& Chiu, 2015; Gillen, Jacquillat \& Odoni, 2016; Bongo \& Ocampo, 2017), na demora na construção e ampliação de pistas, fatores que podem restringir o crescimento do transporte aéreo (Diniz, 2013; Meson-mancha et al., 2019).

\section{Tabela 4}

Pesos obtidos para os subcritérios relacionados ao Lado Ar

\begin{tabular}{|l|l|l|}
\hline \multicolumn{1}{|c}{ Indicador } & \multicolumn{1}{c|}{ Prioridade } & Rank \\
\hline Pista de Pouso e Decolagem (PPD) & $28,63 \%$ & 1 \\
\hline Pistas de Táxi (PTX) & $15,30 \%$ & 2 \\
\hline Número de Classificação de Proteção Existente (NCPE) & $12,10 \%$ & 3 \\
\hline Número de Classificação de Proteção Requerida (NCPR) & $11,40 \%$ & 4 \\
\hline Capacidade de Pouso e Decolagem (slots) (CPD) & $9,40 \%$ & 5 \\
\hline Auxílios de Navegação Aérea (ANA) & $8,20 \%$ & 6 \\
\hline Instrumentos de Navegação Aérea (INA) & $7,40 \%$ & 7 \\
\hline Capacidade de Abastecimento (PAA) & $5,30 \%$ & 8 \\
\hline Disponibilidade de Área para Manutenção de Aeronaves (DMN) & $2,17 \%$ & 9 \\
\hline
\end{tabular}

Fonte: Autores. 
Para avaliação da infraestrutura aeroportuária, relacionada com o Lado Ar (LA), pode-se usar a Equação 2.

$$
\begin{aligned}
& L A=0,2863 P P D+0,153 P T X+0,121 \mathrm{NCPE}+0,114 \mathrm{NCPR}+0,094 \mathrm{CPD}+0,082 \mathrm{ANA}+0,074 \\
& \text { INA + 0,053 PAA + 0,0217 DMN }
\end{aligned}
$$

A Tabela 5 apresenta os dados relativo aos indicadores do Lado Terra, com razão de consistência 9,1\%, sendo que as maiores prioridades estão relacionadas com Área de Processamento de Bagagens, Número de Esteira de Desembarque, Pontes de Embarque/Desembarque, Área da Sala de Embarque, Check-in, Saguão, Canais de Inspeção de Segurança e Facilidade de Conexão. Tais características estão ligadas diretamente à operação de um aeroporto hub, pois são indicadores de suporte ao passageiro que necessitam tanto da estrutura instalada como de funcionários para atendimento e controle dos processos. Ashford et al. (2015) definem o manuseio de bagagens como um elemento fundamental nas operações e fator que detém maior número de reclamações dos clientes, responsabilidade que é tanto da empresa aérea como do aeroporto. Nota-se que as prioridades estabelecidas também possuem pouca variação, principalmente devido a fatores que podem melhorar a eficiência das atividades aeroportuárias, diminuir a espera e oferecer espaço para conforto dos usuários.

\section{Tabela 5}

Pesos obtidos para os subcritérios relacionados ao Lado Terra

\begin{tabular}{|l|l|l|}
\hline \multicolumn{1}{|c|}{$\begin{array}{l}\text { Indicador } \\
\text { Área de processamento de bagagens (bag drop) (APB) }\end{array}$} & $9,6 \%$ & 1 \\
\hline Número de Esteiras de Desembarque (NED) & $8,7 \%$ & 2 \\
\hline Pontes de Embarque/Desembarque (PED) & $8,2 \%$ & 3 \\
\hline Área da Sala de Embarque (SE) & $8,0 \%$ & 4 \\
\hline Check-in compartilhado (CKC) & $8,0 \%$ & 5 \\
\hline Dimensão do Saguão (SG) & $7,8 \%$ & 6 \\
\hline Check-in exclusivo (CKE) & $7,6 \%$ & 7 \\
\hline Canais de Inspeção de Segurança (CIS) & $7,6 \%$ & 8 \\
\hline Facilidade de Conexão (CNX) & $6,3 \%$ & 9 \\
\hline Embarque Remoto (ER) & $5,3 \%$ & 10 \\
\hline Sistema de informação ao passageiro (SIP) & $4,2 \%$ & 11 \\
\hline Disponibilidade de Hotel de Passagem (DHP) & $4,1 \%$ & 12 \\
\hline Praça de Alimentação (PA) & $3,8 \%$ & 13 \\
\hline Disponibilidade de Meio-Fio (MF) & $3,7 \%$ & 14 \\
\hline Mix de Estabelecimentos Comerciais (MEC) & $3,6 \%$ & 15 \\
\hline Área do Estacionamento Comercial (EST) & $3,4 \%$ & 16 \\
\hline
\end{tabular}

Fonte: Autores.

Para avaliação da infraestrutura aeroportuária, relacionada com o Lado Terra (LT), pode-se usar a Equação 3. 
$\mathrm{LT}=0,096 \mathrm{APB}+0,087 \mathrm{NED}+0,082 \mathrm{PED}+0,08 \mathrm{SE}+0,08 \mathrm{CKC}+0,078 \mathrm{SG}+0,076 \mathrm{CKE}+$ $0,076 \mathrm{CIS}+0,063 \mathrm{CNX}+0,053 \mathrm{ER}+0,042 \mathrm{SIP}+0,041 \mathrm{DHP}+0,038 \mathrm{PA}+0,037 \mathrm{MF}+0,036$

$\mathrm{MEC}+0,034 \mathrm{EST}$

A Tabela 6 apresenta os dados relativo aos indicadores referentes aos Serviços de Suporte, com razão de consistência 6,6\%: a maior importância está relacionada com os Modos de acesso, seguida pela Confiabilidade do tempo de acesso, Disponibilidade de Hotéis (distância e a quantidade de leitos). A facilidade e disponibilidade de acesso aos aeroportos gera uma grande preocupação a administradores aeroportuários, pois envolve questões políticas e sociais que podem interferir diretamente no volume de tráfego. A importância desses critérios também pode responder a transformação desse setor em um "Centro de Negócios Multimodal”, como Senguttuvan (2006) propôs, em que o incentivo financeiro nos Serviços de Suporte e principalmente nos Modos de acesso, seriam indispensáveis.

\section{Tabela 6}

Pesos obtidos para os subcritérios relacionados aos Serviços de Suporte

\begin{tabular}{|l|l|l|}
\hline \multicolumn{1}{|c|}{ Indicador } & Prioridade & Rank \\
\hline Modos de acesso (MA) & $45,65 \%$ & 1 \\
\hline Confiabilidade do tempo de acesso (CTA) & $17,35 \%$ & 2 \\
\hline Disponibilidade de Hotéis (distância) (DHD) & $16,80 \%$ & 3 \\
\hline Disponibilidade de Hotéis (leitos) (DHL) & $12,80 \%$ & 4 \\
\hline Disponibilidade de Locais para realização de Eventos (DLE) & $7,40 \%$ & 5 \\
\hline
\end{tabular}

Fonte: Autores.

Na avaliação dos Serviços de Suporte (SS), pode-se usar a Equação 4.

$$
\mathrm{SS}=0,4565 \mathrm{MA}+0,1735 \mathrm{CTA}+0,168 \mathrm{DHD}+0,128 \mathrm{DHL}+0,074 \mathrm{DLE}
$$

O modelo proposto de avaliação remete a necessidade de avaliação de cada característica da infraestrutura aeroportuária e dos serviços de suporte.

\section{Considerações finais}

A partir dos resultados da pesquisa foi possível definir as características técnicas pertinentes a implantação de um hub por determinada empresa aérea, de forma a auxiliar a gestão aeroportuária na definição de prioridades de investimentos e adequação da infraestrutura. Por meio do diagnóstico dos pesos referentes as variáveis abordadas, é possível avaliar medidas no planejamento aeroportuário realizando mudanças que tornem a infraestrutura mais competitiva. 
Sabe-se que o transporte aéreo tem alcançado crescimento elevado e mais pesquisas estão sendo desenvolvidas para melhoria dos serviços e operações, principalmente relacionadas a capacidade. Com este estudo foi possível comprovar a importância do Lado Ar, responsável por comportar ativos destinados a atender aeronaves de grande porte e em uma maior frequência, como é proposto pelo sistema hub and spoke, essa relação também é visualizada pelos subcritérios que obtiveram maior grau de importância, as pistas de circulação, Pista de Pouso e Decolagem (PPD), seguido pelas Pistas de Táxi.

Outra característica atribuída a esse sistema é o aumento da demanda de passageiros, o que significa que as operações no TPS e os serviços que garantem o conforto da chegada e saída dos usuários, também são significativos para o sucesso da implantação de um hub. Nota-se que há uma relação entre o Lado Terra e os Serviços de Suporte quase mínima, visualizada nos resultados de prioridade com variação de apenas 7,55\%. Os indicadores avaliados no Lado Terra obtiveram variações mínimas, com destaque a Área de Processamento de Bagagens e Número de Esteira de Desembarque. Entre os Serviços de Suporte, por outro lado, se destacaram os Modos de acesso, resultado condizente com os argumentos de Button et al. (1999): a região que comporta o hub deve desfrutar dos benefícios do sistema, logo o direito aos modos de acesso deve existir do aeroporto a qualquer ponto da região que o comporta e vice-versa.

A utilização do método AHP mostrou-se apropriada considerando que obteve resultados bastantes coerentes com o que foi analisado da estrutura de aeroportos que comportam este tipo de sistema, o que leva a estudos futuros que busquem quantificar esses componentes com o intuito de selecionar aeroportos que melhor se adequem as expectativas de uma empresa aérea. Ressalta-se que os resultados foram obtidos através de uma pesquisa de opinião aplicada a especialistas na área, os quais foram analisados dentro do próprio método como consistentes. Outras pesquisas também podem ser aprofundadas no intuito de analisar esses pesos obtidos em função da dinâmica competitiva entre aeroportos que pretendem se estabelecer como hub.

\section{Agradecimentos}

Ao CNPq, pela bolsa de produtividade em pesquisa concedida a um dos autores do artigo.

\section{Referências}

Addepalli, S., Pagalday, G., Salonitis K., \& Roy, R. (2018). Socio-economic and demographic factors that contribute to the growth of the civil aviation industry. Procedia Manufacturing. Bremen, 2-9.

Almeida, C. R. de (2011). Low Cost Airlines, Airports and Tourism. The Case of Faro Airport. Ersa 2011, Barcelona, 1-17. 
Almeida, C. R. de, \& Costa, C. (2014). A operação das companhias aéreas nos aeroportos hub \& spoke e nas bases operacionais. Pasos, Portugal, 765-775.

Ashford, N. J., Stanton, H. P. M., Moore C. A., Coutu P., \& Beasleyet J. R. (2015). Operações aeroportuárias: As melhores práticas (3a ed.). Porto Alegre: Bookman.

Bandeira, M., \& Correia, A. (2006). Determinação dos critérios para avaliar o nível de serviço de um aeroporto. Anais do Encontro de Iniciação Científica e Pós-Graduação do ITA, São José dos Campos, SP, Brasil, 12.

Bongo, M. F., \& Ocampo, L. A. (2017). A hybrid fuzzy MCDM approach for mitigating airport congestion: A case in Ninoy Aquino International Airport. Journal of Air Transport Management, 63, 1-16. http://doi.org/10.1016/j.jairtraman.2017.05.004

Borenstein, S. (1989). Hubs and high fares: dominance and market power in the U.S. airline industry. RAND Journal of Economics, 20(3), 344-365.

Button, K. (2002). Debunking some common myths about airport hubs. Journal of Air Transport Management, 8, 177-188.

Button, K., Lall S., Stough R., \& Trice M. (1999). High-technology employment and hub airports. Journal of Air Transport Management, 5(1), 53-59.

Campos, P., Silva J., Pereira A., \& Moreira C. (2010). Logística Aeroportuária: Análises Setoriais e o Modelo de Cidades-Aeroportos (2a ed.). São Paulo: Cengage Learning.

Costa, L. H. G., \& Santos E. (2016). Análise do desempenho operacional dos aeroportos brasileiros na visão dos passageiros. Anais do Congresso de Pesquisa e Ensino em Transportes (ANPET), Rio de Janeiro, RJ, Brasil, 30.

Danesi, A., \& Lupi, M. (2005). Il sistema di trasporto aéreo nazionale italiano: sviluppo della domanda passeggeri ed evoluzion estrutturale della rete. Trasporti \& Territorion, 1, 5-17.

Daniel, J. I. (1995). Congestion Pricing and Capacity of Large Hub Airports: A Bottleneck Model with Stochastic Queues. Econometrica, 63(2), 327-370.

Diniz, R. R. (2013). Dimensionamento de ampliação do aeroporto de Marabá com base em estudo de previsão de demanda aeroportuária. Journal of Transport Literature, 7(1),147-162. http://doi.org/10.1590/s2238-10312013000100009

Franek, J., \& Kresta, A. (2014). Judgment scales and consistency measure in AHP. Procedia Economics and Finance, 12, 164-173. http://doi.org/10.1016/s2212-5671(14)00332-3

Gillen, D., Jacquillat, A., \& Odoni A. R. (2016). Airport demand management: The operations research and economics perspectives and potential synergies. Transportation Research Part A, 94, 495513.

Goepel, K.D. (2018). Implementation of an Online Software Tool for the Analytic Hierarchy Process (AHP-OS). International Journal of the Analytic Hierarchy Process, 10 (3), 469-487. https://doi.org/10.13033/ijahp.v10i3.590

Graham, A. (2014). Managing Airports: An International Perspective (4a ed.). London: Routledge. 
Kidokoro, Y., \& Zhang A. (2018). Airport congestion pricing and cost recovery with side business. Transportation Research Part A: Policy and Practice, 114, 222-236. https://doi.org/10.1016/j.tra.2017.12.003

Lambert, J. M. (1991). The Extended Analytic Hierarchy Decision Method. Mathematical and Computer Modelling, 15(11), 141-151.

Lelles, L. (2001). Mercado do Transporte Aéreo Brasileiro: o Papel do Sistema Hub and spoke. Monografia de Especialização, Universidade de Brasília, Brasília, DF, Brasil.

Liang, M., Delahaye, D., \& Maréchal, P. (2017). Integrated sequencing and merging aircraft to parallel runways with automated conflict resolution and advanced avionics capabilities. Transportation Research Part C: Emerging Technologies, 85, 268-291. http://doi.org/10.1016/j.trc.2017.09.012

Lin, M. H. (2006). Hub-Airport Competition: Connecting time differentiation and concession consumption. Australian Economic Papers, 45(4), 299-317. http://doi.org/10.1111/j.14678454.2006.00295.x

Meson-mancha, S., Lange, T., Koelle, R., \& Carro, I. G. (2019). Assessing the Impact of the Runway System Configuration on Airport Capacity. 2019 Integrated Communications, Navigation And Surveillance Conference (icns), [s.I.], p.1-9, abr. 2019. IEEE. http://dx.doi.org/10.1109/icnsurv.2019.8735166

Mohri, S., Karimi, H., Kordani, A. A., \& Nasrollahi, M. (2018). Airline hub-and-spoke network design based on airport capacity envelope curve: A practical view. Computers \& Industrial Engineering, 125, 375-393. http://doi.org/10.1016/j.cie.2018.09.010

Moreira, B. H. N. (2006). Transporte Aéreo: A Inserção da Região Nordeste Brasileira nas Artérias da Globalização. Dissertação de Mestrado, Universidade Salvador (Unifacs), Salvador, BA, Brasil.

Nosal, K., \& Solecka K. (2014). Application of AHP method for multi-criteria evaluation of variants of the integration of urban public transport. Transportation Research Procedia, 3, 269-278. http://doi.org/10.1016/j.trpro.2014.10.006

Pishdar, M., Ghasemzadeh, F., \& Antuchevičienè, J. (2019). A Mixed Interval Type-2 Fuzzy Best-worst Macbeth Approach to Choose Hub Airport in Developing Countries: Case of Iranian Passenger Airports. Transport, 34 (6), p.639-651. http://doi.org/10.3846/transport.2019.11723

Redondi, R., Malighetti P., \& Paleari, S. (2011). Hub competition and travel times in the world-wide airport network. Transport Geography, 19(6), 1260-1271. https://doi.org/10.1016/j.jtrangeo.2010.11.010

Saaty, R. W. (1987). The Analytic Hierarchy Process-What it is and How it is used. Mathematical Modelling, 9(5), 161-176. https://doi.org/10.1016/0270-0255(87)90473-8

Senguttuvan, P. S. (2006). Economics of the Airport Capacity System in the Growing Demand of Air Traffic - A Global View. Transport research annual forum, New York, 1-29.

Song, W., \& Ma, Y. (2006). Hub-and-Spoke System in Air Transportation and Its Implications to Regional Economic Development: A Case Study of United States. Chinese Geographical Science, 16(3), 211-216. http://doi.org/10.1007/s11769-006-0211-2 
Ssamula, B. (2010). Exploring Multi-criteria Decision Analysis Method as a Tool to Choose Regional Airport Hubs Within Africa. International Journal of Sustainable Development And Planning, 5(2), 83-97.

Torkestani, S. S., Seyedhosseini, S. M., Makui, A., \& Shahanaghi, K. (2018). The reliable design of a hierarchical multi-modes transportation hub location problems (HMMTHLP) under dynamic network disruption (DND). Computers \& Industrial Engineering, 122, 39-86.

http://doi.org/10.1016/j.cie.2018.05.027

Yang, T., \& Chiu, T. (2015). Airline hub-and-spoke system design under stochastic demand and hub congestion. Journal of Industrial And Production Engineering, 33(2), 69-76.

http://dx.doi.org/10.1080/21681015.2015.1107860

Zhang, A., \& Czerny, A. (2012). Airports and airlines economics and policy: An interpretive review of recent research. Economics of Transportation, 1(2), 15-34.

https://doi.org/10.1016/j.ecotra.2012.08.001 